International Journal of Pure and Applied Mathematics

Volume 86 No. 2 2013, 443-455

ISSN: 1311-8080 (printed version); ISSN: 1314-3395 (on-line version)

url: http://www.ijpam.eu

doi: http://dx.doi.org/10.12732/ijpam.v86i2.17

ijpam.eu

\title{
THE ASSOCIATED CLAIM SIZE DISTRIBUTION ARISING IN THE RISK PROCESS WITH RANDOM PREMIUM INCOME
}

\author{
Zhenhua Bao ${ }^{1 \S}$, Yongyi $\mathrm{Fu}^{2}$, Minge $\mathrm{Yin}^{3}$ \\ $1,2,3$ School of Mathematics \\ Liaoning Normal University \\ Dalian, 116029, P.R. CHINA
}

\begin{abstract}
In his paper we consider the associated claim size distribution arising in the risk model with random premium income. We derive conditions under which the aging properties of loss distribution are preserved for the associated claim size distribution. The ordering relationships between the associated claim size distribution and loss distribution for the failure rate function and mean residual lifetime are also investigated.
\end{abstract}

AMS Subject Classification: 62P05, 91B30

Key Words: associated claim size distribution, failure rate function, mean residual lifetime

\section{Introduction}

In the classical Cramér-Lundberg risk model, the number of claims up to time $t$ is assumed to follow a Poisson process $\left\{N_{1}(t), t \geq 0\right\}$ with parameter $\lambda_{1}$. The individual claim sizes $X_{1}, X_{2}, \cdots$, independent of $N_{1}(t)$, are positive independent and identically distributed(i.i.d.) random variables with finite mean $\mu>0$. The surplus of the insurer at time $t$ is

$$
S(t)=u+c t-\sum_{i=1}^{N_{1}(t)} X_{i},
$$

where $u=S(0)$ is the initial surplus and $c$ is a positive real constant corre-

Received: May 13, 2013

(c) 2013 Academic Publications, Ltd.

${ }^{\S}$ Correspondence author 
sponding to the premium income rate. See Asmussen [1] and the references therein for details on this well known model and its generalizations.

In the present paper, we assume the premium income is no longer a linear function of the time but another Poisson process $\left\{N_{2}(t), t \geq 0\right\}$ with parameter $\lambda_{2}$, independent of $\left\{N_{1}(t), t \geq 0\right\}$ and $X_{1}, X_{2}, \cdots$. Where $N_{2}(t)$ denotes the number of the customers up to the time $t$. For simplicity, suppose that the income rate is 1 . That is, the surplus at the time of $t$ is

$$
U(t)=u+N_{2}(t)-\sum_{i=1}^{N_{1}(t)} X_{i},
$$

the readers are referred to Temnov [2], Bao [3] for the study of related model.

Throughout, denote by $N$ the set of nature numbers and $N_{+}=N-\{0\}$. We assume that

$$
\lambda_{2}>\lambda_{1} \mu
$$

to ensure the positive safety loading condition as the classical case. Also, $u$ is a non-negative integer and $X_{1}, X_{2}, \cdots$ is a sequence of integer-valued random variables having common cumulative distribution function(c.d.f.) $F(x)=1-$ $\bar{F}(x)$ and probability function(p.f) $f(x), x \in N_{+}$and $f(0)=0$ to avoid noncontribution claims. We assume that $\bar{F}(x)>0$ for all $x \in N$. See Picard and Lefevre [4] and the references therein for the study of the risk model with discrete claim size distribution.

Let $T=\inf \{t \geq 0: U(t)<0\}$ be the time of ruin. Note that if ruin occurs, $|U(T)|$ is the deficit at ruin and $U(T-)$ is the surplus immediately prior to ruin. Denote by

$$
m(u)=E\left[e^{-\delta T} \omega(U(T-),|U(T)|) I(T<\infty) \mid U(0)=u\right]
$$

the expected discounted penalty function, which was first introduced by Gerber and Shiu [5]. Here, $\delta \geq 0$ is the discounted factor, $\omega\left(u_{1}, u_{2}\right): N \times N_{+} \rightarrow N$ and $I(\cdot)$ is the indicator function. Note that the symbol $m(u)$ does not exhibit the dependence on the parameter $\delta$ for convenience. The function $m(u)$ is useful for unifying the results in connection with joint and marginal distributions of $T, U(T-)$ and $|U(T)|$, see Gerber and Shiu $[5,6]$ for details.

The analysis in this paper depends upon reliability based properties and equilibrium distributions associated with a given discrete c.d.f.. In particular, the equilibrium c.d.f. of $X_{1}$ is defined as $F_{1}(x)=1-\bar{F}_{1}(x)$ with p.f. $f_{1}(x)=\bar{F}(x-1) / \mu, x \in N_{+}$, and in the present context $F_{1}(x)$ may be interpreted as the c.d.f. of the amount of the drop in the surplus level, given 
that there is a drop(e.g., Bowers et al. [7]). The c.d.f. $F(x)$ is said to be discrete decreasing(increasing) failure rate or D-DFR(D-IFR) if $\frac{\bar{F}(n+1)}{\bar{F}(n)}$ is nondecreasing (nonincreasing) in $n \in N$. Larger classes of distributions are the new worse(better) than used or D-NWU(D-NBU) class with $\bar{F}(m+n) \geq(\leq$ $\bar{F}(m) \bar{F}(n)$ for all $m, n \in N$, the D2-NWU(D2-NBU) class for which $F_{1}(x)$ is D-NWU(D-NBU), and the new worse(better) than used in convex ordering or D-NWUC(D-NBUC) class where $\bar{F}_{1}(m+n) \geq(\leq) \bar{F}_{1}(m) \bar{F}(n)$ for all $m, n \in N$.

The discrete failure rate of $F(x)$ may be defined as

$$
h_{F}(n)=P\{X=n \mid X \geq n\}=\frac{f(n)}{f(n)+\bar{F}(n)}, \quad n \in N .
$$

It is easy to see that

$$
\frac{\bar{F}(n+1)}{\bar{F}(n)}=1-h_{F}(n+1), \quad n \in N,
$$

from (4) we know that $F(x)$ is D-DFR(D-IFR) if $h_{F}(n)$ is nonincreasing (nondecreasing) in $n \in N_{+}$. Also, the mean residual lifetime of $F(x)$ is defined by

$$
r_{F}(n)=E(X-n \mid X>n)=\frac{\sum_{k=n}^{\infty} \bar{F}(k)}{\bar{F}(n)}=r_{F}(0) \frac{\bar{F}_{1}(n)}{\bar{F}(n)}, \quad n \in N .
$$

Another class larger than D-DFR(D-IFR) is the increasing (decreasing) mean residual lifetime or D-IMRL(D-DMRL) class for which $r_{F}(n)$ is nondecreasing (nonincreasing) for $n \in N$. Similarly, $F(x)$ is the new worse(better) than used in expectation or D-NWUE(D-NBUE) if $\bar{F}_{1}(n) \geq(\leq) \bar{F}(n)$, or equivalently, $r_{F}(n) \geq(\leq) r_{F}(0)$ by (5). See Fagiuoli and Pellerey [8], Willmot and Lin [9], Hu et al. [10] and the references therein for further details on these classifications.

The rest of this paper is organized as follows: In Section 2, we discuss the defective renewal equation satisfied by the Gerber-Shiu expected discounted penalty function $m(u)$. In Section 3, we investigate some distributional properties of the associated claim size distribution in detail. Using the reliability based properties for the discrete c.d.f., we show that some aging properties are preserved for the associated claim size distribution. Further, we give the failure rate(resp. the mean residual lifetime) ordering between the associated claim size distribution and the loss distribution. 


\section{Defective Renewal Equation for $m(u)$}

Since the pioneering work of Gerber-Shiu [5], it is desirable to give the renewal equation satisfied by the expected discounted penalty function $m(u)$ in risk theory. To study the the discounted penalty function, many authors employ the efficient method of conditioning on the claim occurrence during the first time period. The efficiency of it is mostly due to the independent increment property of the risk processes. It is obvious that there are similarities between the classical Poisson model and the risk model (1). However, the present approach in deriving the renewal equation for Poisson model involves differencing, the technique must be modified to obtain a discrete defective renewal equation for the risk model with discrete claim size distribution. The following result was obtained by Bao [3], which can be regarded as an analog of the defective renewal equation satisfied by the Gerber-Shiu function in the classical Poisson risk model (e.g., Gerber and Shiu [5]).

In this section, we use the curly capital letters to denote the corresponding generating functions throughout entire paper and we adopt the convention that $\sum_{a}^{b}=0$ when $b<a$.

Proposition 2.1. (see [3]) For $u \in N$, the Gerber-Shiu expected discounted penalty function $m(u)$ satisfies the following renewal equation:

$$
m(u)=b(\rho) \sum_{i=1}^{u} m(u-i) \eta(i)+b(\rho) \zeta(u),
$$

its initial value $m(0)$ is

$$
m(0)=\frac{\lambda_{1} \rho}{\lambda_{2}} \mathcal{W}(\rho)
$$

where

$$
b(\rho)=\frac{\lambda_{1}}{\lambda_{1}+\lambda_{2}+\delta-\lambda_{1} \mathcal{F}(\rho)},
$$

and $\rho=\rho(\delta)$ is the unique positive root in the interval $\left(\frac{\lambda_{2}}{\lambda_{1}+\lambda_{2}+\delta}, \frac{\lambda_{1}+\lambda_{2}}{\lambda_{1}+\lambda_{2}+\delta}\right]$ to the equation

$$
\begin{array}{r}
\lambda_{1} \rho \mathcal{F}(\rho)=\left(\lambda_{1}+\lambda_{2}+\delta\right) \rho-\lambda_{2} . \\
\eta(i)=\sum_{k=i}^{\infty} \rho^{k-i} f(k), i \in N_{+} \text {and } \zeta(u)=\sum_{j=u}^{\infty} \rho^{j-u} \omega(j), \\
\omega(j)=\sum_{k=j+1}^{\infty} \omega(j, k-j) f(k), \quad j \in N .
\end{array}
$$


It has been proved in Bao [3] that the renewal equation (6) is defective for all $\delta \geq 0$. Let $j \in N_{+}$, by reversing the order of summation we obtain

$$
\begin{aligned}
\sum_{i=j+1}^{\infty} \sum_{k=i}^{\infty} \rho^{k-i} f(k) & =\sum_{i=j+1}^{\infty} \sum_{k=0}^{\infty} \rho^{k} f(k+i) \\
& =\sum_{k=0}^{\infty} \rho^{k} \sum_{i=j+1}^{\infty} f(k+i) \\
& =\sum_{k=0}^{\infty} \rho^{k} \bar{F}(k+j) .
\end{aligned}
$$

Put $j=0$ in $(7)$ to obtain

$$
\sum_{i=1}^{\infty} \sum_{k=i}^{\infty} \rho^{k-i} f(k)=\sum_{k=0}^{\infty} \rho^{k} \bar{F}(k) \equiv D .
$$

From the equation (7), we know that

$$
g(x)=\frac{\sum_{i=x}^{\infty} \rho^{i-x} f(i)}{D}, \quad x \in N_{+}
$$

is a proper p.f.. Also, from (8) the associated c.d.f. $G(x)=1-\bar{G}(x)$ is given by

$$
\bar{G}(x)=\frac{\sum_{k=0}^{\infty} \rho^{k} \bar{F}(k+x)}{D}, \quad x \in N .
$$

The c.d.f. $G(x)$ is called the associated claim size distribution in the risk theory context, many of whose useful properties are now given.

\section{The Associated Claim Size Distribution}

The c.d.f. $G(x)$ given by (10) has many useful properties for the present application. Clearly, when $\rho=1$ which is the case when $\delta=0$, from (9) one obtains $g(x)=f_{1}(x)$, i.e., $G(x)=F_{1}(x), x \in N_{+}$. We now show that $G(x)$ is of the same form as $F_{1}(x)$ in the following example.

Example 3.1. Suppose that the sequence $\left\{q_{1}, q_{2}, \cdots, q_{r}\right\}$ satisfy $q_{1}+q_{2}+$ $\cdots+q_{r}=1$ and $f(k)=\sum_{i=1}^{r} q_{i}\left(1-\phi_{i}\right) \phi_{i}^{k-1}, k \in N_{+}$, where $0<\phi_{i}<1, i=$ $1,2, \cdots, r$. If $q_{i}>0$ for all $i$ then $f(k)$ is a mixture of geometric distributions 
whereas if $q_{i}<0$ for some $i$ then $f(k)$ is termed a combination of geometric distributions. Then

$$
\bar{F}(x)=\sum_{k=x+1}^{\infty} f(k)=\sum_{i=1}^{r} q_{i} \phi_{i}^{x}, \quad x \in N,
$$

and from (11)

$$
\sum_{k=x}^{\infty} \rho^{k} \bar{F}(k)=\sum_{i=1}^{r} \frac{q_{i}}{1-\rho \phi_{i}}\left(\rho \phi_{i}\right)^{x} .
$$

Thus, from (10) and (12) we have

$$
\bar{G}(x)=\sum_{i=1}^{r} q_{i}^{*} \phi_{i}^{x}, \quad x \in N
$$

where

$$
q_{i}^{*}=\frac{q_{i} /\left(1-\rho \phi_{i}\right)}{\sum_{j=1}^{r} q_{j} /\left(1-\rho \phi_{j}\right)}, \quad i=1,2, \cdots, r .
$$

Consequently, $G(x)$ is also a mixture or combination of geometric distributions. Two special cases: $r=1$ and $r=2$ are of certain importance. When $r=1$, then $F(x)$ is geometric distribution and one obtains $G(x)=F_{1}(x)=F(x)$, i.e., the associated claim size distribution remains unchanged. When $r=2$, we have a mixture or combination of two geometric distributions. The resulting associated claim size distribution is again a mixture or combination of two geometric distributions with new weights.

Now we begin to discuss the aging properties of $G(x)$. It is easy to see that the failure rate of $G(x)$ is

$$
h_{G}(n)=\frac{g(n)}{\bar{G}(n-1)}=\frac{1-\rho}{\rho}\left\{\frac{1}{\gamma(n-1)}-1\right\}, \quad n \in N_{+},
$$

where

$$
\gamma(n)=(1-\rho) \sum_{k=0}^{\infty} \rho^{k} \frac{\bar{F}(k+n)}{\bar{F}(n)}, \quad n \in N .
$$

In fact, for any $n \in N$

$$
\sum_{k=0}^{\infty} \rho^{k} \bar{F}(k+n)=\sum_{k=n}^{\infty} \rho^{k-n} \bar{F}(k)=\sum_{k=n}^{\infty} \rho^{k-n} \sum_{j=k+1}^{\infty} f(j)
$$




$$
\begin{aligned}
& =\rho^{-n} \sum_{j=n+1}^{\infty} f(j) \sum_{k=n}^{j-1} \rho^{k} \\
& =\frac{1}{1-\rho}\left[\bar{F}(n)-\rho \sum_{j=n+1}^{\infty} \rho^{j-(n+1)} f(j)\right],
\end{aligned}
$$

then (14) follows from (16), (9) and (10) after rearranging.

Suppose that the equilibrium c.d.f. of $G(x)$ is $G_{1}(x)$ with p.f. $g_{1}(x), x \in N_{+}$. Then for $x \in N$

$$
\begin{aligned}
\sum_{k=x}^{\infty} \bar{G}(k) & =\frac{1}{D} \sum_{k=x}^{\infty} \sum_{j=k}^{\infty} \rho^{j-k} \bar{F}(j)=\frac{1}{D} \sum_{k=x}^{\infty} \sum_{m=0}^{\infty} \rho^{m} \bar{F}(m+k) \\
& =\frac{1}{D} \sum_{m=0}^{\infty} \rho^{m} \sum_{k=x}^{\infty} \bar{F}(m+k) \\
& =\frac{1}{D} \sum_{m=0}^{\infty} \rho^{m} \sum_{j=m+x}^{\infty} \bar{F}(j) \\
& =\frac{\mu}{D} \sum_{m=0}^{\infty} \rho^{m} \sum_{j=m+x}^{\infty} f_{1}(j+1) \\
& =\frac{\mu}{D} \sum_{m=0}^{\infty} \rho^{m} \bar{F}_{1}(m+x) .
\end{aligned}
$$

Let $x=0$ in (17), we obtain the mean of $G(x)$

$$
\sum_{k=0}^{\infty} \bar{G}(k)=\frac{\mu}{D} \sum_{m=0}^{\infty} \rho^{m} \bar{F}_{1}(m) .
$$

By (17) and (18), the equilibrium c.d.f. of $G(x)$ is given by

$$
\bar{G}_{1}(x)=\frac{\sum_{m=0}^{\infty} \rho^{m} \bar{F}_{1}(m+x)}{\sum_{m=0}^{\infty} \rho^{m} \bar{F}_{1}(m)} .
$$

On the other hand, by (4) and (5) we obtain

$$
h_{G_{1}}(n+1)=\frac{g_{1}(n+1)}{\bar{G}_{1}(n)}=\frac{\bar{G}(n)}{\sum_{k=n}^{\infty} \bar{G}(k)}=\frac{1}{r_{G}(n)}, \quad n \in N .
$$

By analogy with (16) we have

$$
\sum_{m=0}^{\infty} \rho^{m} \bar{F}_{1}(m+n)=\sum_{k=n}^{\infty} \rho^{k-n} \bar{F}_{1}(k)
$$




$$
=\frac{1}{1-\rho}\left[\bar{F}_{1}(n)-\rho \sum_{j=n}^{\infty} \rho^{j-n} f_{1}(j+1)\right],
$$

by (20) and (21), we can rewritten the mean residual lifetime $r_{G}(n)$ as

$$
\frac{1}{r_{G}(n)}=\frac{1-\rho}{\rho}\left\{\frac{1}{\gamma_{1}(n)}-1\right\}, \quad n \in N,
$$

where

$$
\gamma_{1}(n)=(1-\rho) \sum_{k=0}^{\infty} \rho^{k} \frac{\bar{F}_{1}(k+n)}{\bar{F}_{1}(n)}, n \in N .
$$

Also, by (21) we have

$$
\begin{aligned}
\sum_{k=n}^{\infty} \rho^{k-n} \frac{\bar{F}(k)}{\mu} & =\sum_{k=n}^{\infty} \rho^{k-n} f_{1}(k+1) \\
& =\frac{1}{\rho} \bar{F}_{1}(n)-\frac{1-\rho}{\rho} \sum_{k=n}^{\infty} \rho^{k-n} \bar{F}_{1}(k),
\end{aligned}
$$

by (24), the equation (15) yields

$$
\begin{aligned}
\gamma(n) & =\frac{(1-\rho) \mu}{\bar{F}(n)} \sum_{k=n}^{\infty} \rho^{k-n} \frac{\bar{F}(k)}{\mu} \\
& =\frac{(1-\rho) \mu \bar{F}_{1}(n)}{\rho \bar{F}(n)}\left\{1-(1-\rho) \sum_{k=0}^{\infty} \rho^{k} \frac{\bar{F}_{1}(k+n)}{\bar{F}_{1}(n)}\right\} .
\end{aligned}
$$

From the equations (5) and (23), (25) may be restated as

$$
\gamma(n)=\frac{(1-\rho)}{\rho} r_{F}(n)\left\{1-\gamma_{1}(n)\right\}, \quad n \in N .
$$

We may now prove the following theorem.

Theorem 3.1. The following class implications hold.

1. If $F(x)$ is $D$-DFR(D-IFR) then $G(x)$ is $D$-DFR(D-IFR).

2. If $F(x)$ is $D$-IMRL $(D-D M R L)$ then $G(x)$ is $D$ - $I M R L(D-D M R L)$.

3. If $F(x)$ is D2-NWU(D2-NBU) then $G(x)$ is D-NWUE(D-NBUE).

Proof. If $F(x)$ is D-DFR(D-IFR), then $\frac{\bar{F}(n+1)}{\bar{F}(n)}$ is nondecreasing (nonincreasing) in $n$, implying that $\frac{\bar{F}(k+n)}{\bar{F}(n)}$ is nondecreasing (nonincreasing) in $n$ for 
fixed $k$. From (30) that $\gamma(n)$ is also nondecreasing (nonincreasing) in $n$, and (14) that $h_{G}(n)$ is nonincreasing (nondecreasing) in $n$, proving 1.

Also, from the relationship (20) we know that $F(x)$ is D-IMRL(D-DMRL) is equivalent to $F_{1}(x)$ is D-DFR(D-IFR), and so $\frac{\bar{F}_{1}(k+n)}{\bar{F}_{1}(n)}$ is nondecreasing (nonincreasing) in $n$ for fixed $k$, implying from (23) that $\gamma_{1}(n)$ is nondecreasing (nonincreasing) in $n$ and (22) that $r_{G}(n)$ is nondecreasing (nonincreasing) in $n$, proving 2 .

Now turning to 3 . Note that if $F(x)$ is D2-NWU(D2-NBU), then we have $\bar{F}_{1}(k+n) \geq(\leq) \bar{F}_{1}(k) \bar{F}_{1}(n)$. And from (23) we obtain

$$
\gamma_{1}(n) \geq(\leq)(1-\rho) \sum_{k=0}^{\infty} \rho^{k} \bar{F}_{1}(k)=\gamma_{1}(0) .
$$

However, this implies from (22) that

$$
\frac{1}{r_{G}(n)} \leq(\geq) \frac{1-\rho}{\rho}\left\{\frac{1}{\gamma_{1}(0)}-1\right\}=\frac{1}{r_{G}(0)},
$$

i.e.

$$
r_{G}(n) \geq(\leq) r_{G}(0)
$$

Then 3 is proved.

Along the same lines, the failure rate properties for $G(x)$ are now given.

Theorem 3.2. For $n \in N_{+}$, the following failure rate implications hold.

1. If $F(x)$ is $D$-DFR(D-IFR) then $h_{G}(n) \leq(\geq) h_{F}(n)$.

2. If $F(x)$ is $D$-IMRL $(D-D M R L)$ then $h_{G}(n) \geq(\leq) h_{F_{1}}(n)$.

3. If $F(x)$ is $D-N W U(D-N B U)$ then $h_{G}(n) \leq(\geq) h_{G}(1)$.

Proof. To prove 1 , note that for fixed $k \in N_{+}$

$$
\begin{aligned}
& \frac{\bar{F}(k+n)}{\bar{F}(n)} \\
= & \frac{\bar{F}(n+1)}{\bar{F}(n)} \frac{\bar{F}(n+2)}{\bar{F}_{1}(n+1)} \cdots \frac{\bar{F}(n+k)}{\bar{F}(n+k-1)} \\
= & {\left[1-h_{F}(n+1)\right]\left[1-h_{F}(n+2)\right] \cdots\left[1-h_{F}(n+k)\right] . }
\end{aligned}
$$

If $F(x)$ is D-DFR(D-IFR), then $h_{F}(n)$ is nonincreasing (nondecreasing) in $n \in$ $N_{+}$, by (28) we have

$$
\frac{\bar{F}(k+n)}{\bar{F}(n)} \geq(\leq)\left[1-h_{F}(n+1)\right]^{k} .
$$


(15) and (29) imply that

$$
\gamma(n) \geq(\leq)(1-\rho) \sum_{k=0}^{\infty} \rho^{k}\left[1-h_{F}(n+1)\right]^{k}=\frac{1-\rho}{1-\rho\left(1-h_{F}(n+1)\right)}
$$

from (14) and (30) that $h_{G}(n) \leq(\geq) h_{F}(n)$.

The proof of 2 is similar. For fixed $k \in N_{+}$, by (20)

$$
\begin{aligned}
& \frac{\bar{F}_{1}(k+n)}{\bar{F}_{1}(n)} \\
= & {\left[1-h_{F_{1}}(n+1)\right]\left[1-h_{F_{1}}(n+2)\right] \cdots\left[1-h_{F_{1}}(n+k)\right] } \\
= & {\left[1-\frac{1}{r_{F}(n)}\right]\left[1-\frac{1}{r_{F}(n+1)}\right] \cdots\left[1-\frac{1}{r_{F}(n+k-1)}\right] . }
\end{aligned}
$$

If $F(x)$ is D-IMRL(D-DMRL), then $r_{F}(n)$ is nondecreasing (nonincreasing) in $n$, from (31) that

$$
\frac{\bar{F}_{1}(k+n)}{\bar{F}_{1}(n)} \geq(\leq)\left[1-\frac{1}{r_{F}(n)}\right]^{k} .
$$

(23) and (32) imply that

$$
\gamma_{1}(n) \geq(\leq) \frac{(1-\rho) r_{F}(n)}{(1-\rho) r_{F}(n)+\rho}
$$

From (26) and (33) that

$$
\gamma(n) \leq(\geq) \frac{(1-\rho) r_{F}(n)}{(1-\rho) r_{F}(n)+\rho}
$$

(14) and (34) imply that $h_{G}(n) \geq(\leq) \frac{1}{r_{F}(n-1)}=h_{F_{1}}(n)$, proving 2 .

Now turning to (3). If $F(x)$ is D-NWU(D-NBU), then we have $\bar{F}(k+n) \geq$ $(\leq) \bar{F}(k) \bar{F}(n)$ for fixed $k$. And from (15) we obtain

$$
\gamma(n) \geq(\leq)(1-\rho) \sum_{k=0}^{\infty} \rho^{k} \bar{F}(k)=\gamma(0)
$$

Thus, from (14) we know that $h_{G}(n) \leq(\geq) h_{G}(1)$, then 3 is proved.

We remark that part 1 of theorem 3.2 is a failure rate ordering between $G(x)$ and $F(x)$, and 2 between $G(x)$ and $F_{1}(x)$. The mean residual lifetime is now considered. 
Theorem 3.3. For $n \in N$, the following mean residual lifetime implications hold.

1. If $F(x)$ is $D 2-N W U(D 2-N B U)$ then $r_{G}(n) \geq(\leq) r_{G}(0)$.

2. If $F(x)$ is $D-I M R L(D-D M R L)$ then $r_{G}(n) \geq(\leq) r_{F}(n)$.

3. If $F(x)$ is $D$-NWUC $(D-N B U C)$ then $r_{G}(n) \geq(\leq) \frac{1}{h_{G}(1)}$.

4. If $r_{F}(n) \geq(\leq) r$ where $1 \leq r<\infty$, then $r_{G}(n) \geq(\leq) r$.

Proof. Part 1 follows from (27), part 2 follows from (22) and (33) directly.

If $F(x)$ is D-NWUC(D-NBUC) then $\bar{F}_{1}(k+n) \geq(\leq) \bar{F}_{1}(n) \bar{F}(k)$, implying from (19) that

$$
\gamma_{1}(n) \geq(\leq)(1-\rho) \sum_{k=0}^{\infty} \rho^{k} \bar{F}(k)=\gamma(0) .
$$

Therefore, from (14), (22) and (36) we have

$$
\frac{1}{r_{G}(n)} \leq(\geq) h_{G}(1)
$$

i.e., $r_{G}(n) \geq(\leq) \frac{1}{h_{G}(1)}$.

To prove 4 , by (31) we have

$$
\frac{\bar{F}_{1}(k+n)}{\bar{F}_{1}(n)} \geq(\leq)\left(1-\frac{1}{r}\right)^{k},
$$

(23) and (37) imply that

$$
\gamma_{1}(n) \geq(\leq) \frac{(1-\rho) r}{(1-\rho) r+\rho},
$$

then by (22) and (38) we have $r_{G}(n) \geq(\leq) r$.

Stochastic bounds are now considered.

Theorem 3.4. For The following Stochastic implications hold.

1. $\bar{G}(x) \leq \bar{F}_{1}(x) / \mathcal{F}_{1}(\rho), x \in N$.

2. If $F(x)$ is $D-N W U(D-N B U)$ then $\bar{G}(x) \geq(\leq) \bar{F}(x)$.

3. If $F(x)$ is D2-NWU(D2-NBU) then $\bar{G}(x) \leq(\geq) \bar{F}_{1}(x)$.

Proof. Part 2 follows easily from $(10)$ and $\bar{F}(m+n) \geq(\leq) \bar{F}(m) \bar{F}(n), m, n \in$ $N$. Now we show the following equation holds:

$$
\bar{F}_{1}(x)=\left(1-\mathcal{F}_{1}(\rho)\right) \bar{G}_{1}(x)+\mathcal{F}_{1}(\rho) \bar{G}(x) .
$$


As mentioned in the beginning of this section, (39) holds for $\rho=1$. If $\rho<1$, one has easily that

$$
\mathcal{F}_{1}(\rho)=\sum_{k=1}^{\infty} \rho^{k} f_{1}(k)=\frac{\rho D}{\mu} .
$$

On the other hand, by (17) we have for $x \in N$

$$
\begin{aligned}
\sum_{m=0}^{\infty} \rho^{m} \bar{F}_{1}(m+x) & =\frac{1}{\mu} \sum_{k=x}^{\infty} \sum_{j=k}^{\infty} \rho^{j-k} \bar{F}(j) \\
& =\frac{1}{\mu} \sum_{j=x}^{\infty} \rho^{j} \bar{F}(j) \sum_{k=x}^{j} \rho^{-k} \\
& =\frac{\rho \sum_{j=x}^{\infty} \rho^{j-x} \bar{F}(j)-\sum_{j=x}^{\infty} \bar{F}(j)}{\mu(\rho-1)},
\end{aligned}
$$

by (41), the equilibrium distribution $G_{1}(x)$ can be restated as

$$
\bar{G}_{1}(x)==\frac{\rho \sum_{j=x}^{\infty} \rho^{j-x} \bar{F}(j)-\sum_{j=x}^{\infty} \bar{F}(j)}{\rho D-\mu} .
$$

then (39) follows from (10), (40) and (42) after some algebras.

From (39), we obtain $\bar{F}_{1}(x) \geq \mathcal{F}_{1}(\rho) \bar{G}(x)$, proving 1 .

To prove 3, note that if $F(x)$ is D2-NWU(D2-NBU) then from theorem 3.1, $G(x)$ is D-NUUE(D-NBUE), i.e., $\bar{G}_{1}(x) \geq(\leq) \bar{G}(x)$, and from (39)

$$
\bar{F}_{1}(x) \geq(\leq)\left(1-\mathcal{F}_{1}(\rho)\right) \bar{G}(x)+\mathcal{F}_{1}(\rho) \bar{G}(x)=\bar{G}(x),
$$

proving 3 .

\section{Acknowledgements}

This work was supported by National Natural Science Foundation of China (11001114) and Program for Liaoning Excellent Talents in University (LJQ201 $1113)$.

\section{References}

[1] S. Asmussen, Ruin Probabilities, Word Scientific, Singapore (2000). 
[2] G. Temnov, Risk process with random income, J. Math. Sci., 123 (2004), 3780-3794.

[3] Z. Bao, The expected discounted penalty at ruin in the risk process with random income, Appl. Math. Comput., 179 (2006), 559-566.

[4] Ph. Picard, C. Lefevre, The moments of ruin time in the classical risk model with discrete claim size distribution, Insurance Math. Econom., 23 (1998), 157-172.

[5] H.U. Gerber, E.S.W. Shiu, On the time value of ruin, N. Am. Actuar. J., 2 (1998), 48-78.

[6] H.U. Gerber, E.S.W. Shiu, The joint distribution of the time of ruin, the surplus immediately before ruin and the deficit at ruin, Insurance Math. Econom., 219 (1997), 129-137.

[7] N. Bowers et al., Actuarial Mathematics, Society of Actuaries, Ttasca, IL (1997).

[8] E. Fagiuoli, F. Pellerey, Preservation of certain classes of life distributions under Poission shock models, J. Appl. Probab., 31 (1994), 458-465.

[9] G. E. Willmot, X. Lin, Lundburg Approximations for Compound Poisson Distributions with Insurance Applications, Springer-Verlag, New York (2001).

[10] T. Z. Hu, M. Ma and A. K. Nanda, Moment inequalities for discrete ageing families, Commun. Statist. - Theory and Methods, 32 (2003), 61-90. 
